\title{
Molecular Characterization of Mitochondrial Genome From Trichostrongylus Species (Nematoda: Trichostrongylidae) in Northern Iran
}

Meysam Sharifdini

Guilan University of Medical Sciences

Elham Hajialilo ( $\sim$ e.hajialilo@gmail.com )

Qazvin University of Medical Sciences https://orcid.org/0000-0003-2159-4066

Research

Keywords: Trichostrongylus, Cox1, Phylogenetic analysis, Guilan, Iran

Posted Date: December 29th, 2020

DOI: https://doi.org/10.21203/rs.3.rs-134785/v1

License: (c) (1) This work is licensed under a Creative Commons Attribution 4.0 International License.

Read Full License 


\section{Abstract}

Background: The members of Trichostrongylus spp. are gastrointestinal nematodes of ruminants with worldwide distribution. The nematodes are considered as major health challenges especially in endemic regions of Iran. Several species of the parasite are reported from humans and animals in the country. Frequently, molecular analyses for identification of different species focus on nuclear regions and there is lack of information regarding the mitochondrial genes of Trichostrongylus spp. Therefore, the aim of the present study was to identify Trichostrongylus species by molecular analysis of mitochondrial gene in Guilan province, northern Iran.

Methods: The nematodes were collected from the abomasum contents of sheep, goats and cattle. Morphological survey was performed for initial screening. Total DNA was extracted, and the partial region of cytochrome c oxidase subunit I (Cox1) gene was amplified and sequenced. Genetic diversity was calculated and phylogenetic analysis of the nucleotide sequence data was conducted.

Results: Three species of Trichostrongylus including T. colubriformis, T. vitrines and T. axei were identified by morphological characterizations. The genetic divergence within the species in the present study was observed for $T$. axei $(0-2.5 \%)$, T. colubriformis $(0.77 \%)$ and $T$. vitrinus $(0 \%)$. The mean interspecies difference between the Trichostrongylus species obtained in this study was $14.4-15.4 \%$.

Conclusions: The Cox 1 sequences of members of the Trichostrongylus spp. are highly variable and this could be used as a valuable measure for achieving a proper assessment of biodiversity. Sequence data generation from additional species of Trichostrongylus will be needed to reconstruct the phylogenetic relationships of this genus of nematodes.

\section{Background}

Trichostrongylus nematodes are highly prevalent and considered as gastrointestinal parasite pathogens among ruminants with a worldwide distribution $[1,2]$. Trichostrongylus spp. usually infect human by ingesting the infective-stage larvae [1]. Clinical symptoms of humans are mild, though in some patients gastrointestinal signs and eosinophilia may occur [3,4]. These nematodes are major health challenges, causing reduced animal production or even death of the infected animal in severe infection, as well as economic burden with the cost of treatment, considered as a problem especially in developing countries $[5,6]$. Several species of the parasite have been reported from herbivores with approximately 12 species identified in humans [2, 7]. Also, the frequency of Trichostrongylus from human and animal hosts has been repeatedly reported in Iran $[8,9,10,11]$. Ruminant infection was reported from Isfahan, Khuzestan, Mazandaran, Kermanshah, Hormozgan and West Azerbaijan provinces, with human infections found in Khuzestan, Isfahan, Tehran, Hormozgan, Kermanshah, Mazandaran, Guilan, Sistan \& Baluchestan, and West Azerbaijan provinces $[2,12,13,14,15]$.

Identification of the parasite species could be useful in preventing and controlling the disease. According to the morphological futures reported in previous studies from Iran, several species of nematodes have 
been identified in human including T. orientalis, T. vitrinus, T. axei, T. colubriformis, T. probolurus, T. skrjabini, T. capricola, and T. lerouxi $[8,9,14]$. In recent years, some studies clarified the human infections with $T$. vitrinus, $T$. axei, T. colubriformis and T. longispicularis species in endemic areas of northern Iran with $T$. colubriformis considered as a predominant species $[2,12,16,17]$. Infection with various species of Trichostrongylus including $T$. colubriformis $[10,11,14,18,19]$, $T$. vitrinus $[10,11,14,18,19], T$. axei [14], $T$. capricola [10, 14], T. probolurus [10, 11, 14, 18, 19], T. longispicularis [10], T. orientalis [14], T. lerouxi [20], T. skrjabini [14] and T. hamatus [18] were reported in different herbivores such as sheep [10,11, 14], goat $[10,14]$, cattle $[10,14]$, buffalo $[10,14]$ and camel $[18,19]$ in most parts of Iran. The predominant species of Trichostrongylus among different herbivores are T. colubriformis, T. vitrinus, and $T$. axei found in most parts of the country [14]. Also, T. colubriformis, T. vitrines, and T. axei were common species among different herbivores in most parts of the country [14].

There is a tremendous diversity of the nematodes in the country $[10,21]$ however the molecular approaches, currently available and easily applicable, could accurately identify these species. Molecular studies based on internal transcribed spacer (ITS) and 28S regions of ribosomal DNA were applied for genetic variation and phylogenetic analysis of Trichostrongylina [16, 22, 23, 24]. Frequently, many studies focus on ITS2 for analysis of genetic variation, species detection, and phylogenetic relationships $[2,16$, 21]. Mitochondrial ( $\mathrm{mt}$ ) genomes could present valuable information. Mt genomes are conserved and present large amounts of sequence data in the organisms, therefore mtDNA are used for evolutionary analyses, taxonomy, population genetics, and systematics studies $[25,26,27]$. There are few studies that have investigated the mitochondrial gene of Trichostrongylidae family, in which the mtDNA of the species of Marshallagia marshalli, Haemonchus placei, Haemonchus contortus, T. vitrinus, T. axei, Ostertagia trifurcate, and Teladorsagia circumcincta were evaluated for phylogenetic relationship and species identification $[25,28,29,30,31,32,33]$. Taxonomy studies of the nematodes based on sequences of coding mitochondrial genes are more accurate than non-coding ribosomal genes. Meanwhile, the mitochondrial genomes are considered as suitable markers for population evolution studies [31, 32], while the studies targeting the mtDNA for identification of Trichostrongylidae are very limited worldwide with even no single study on mitochondrial gene of the nematodes reported from Iran, so, the present study focused on molecular phylogenetic analysis based on cytochrome c oxidase subunit I (Cox1) mitochondrial gene of Trichostrongylus species in northern Iran.

\section{Methods}

\section{Sample collection and morphological identification}

This study was conducted in Guilan province, located in the littoral of Caspian Sea, northern Iran. The nematodes were collected from abomasum of sheep, goats, and cattle in the abattoir of the town of Talesh, the center of animal husbandry in Guilan province (Fig 1). The Trichostrongylidae family members were isolated by washing the abomasum content followed by passing through the 20,40 , and 100 mesh screens. The helminths captured on mesh screens were examined under stereomicroscope. 
Morphological features were evaluated after cleaning the worms with normal saline and lactophenol. The samples were preserved in $70 \%$ ethanol at room temperature until used [34].

\section{DNA extraction and PCR amplification}

Male parasites were isolated for DNA extraction. Total genomic DNA was extracted from one male worm of each species of trichostrongyloid nematodes from every kind of livestock using a commercial DNA extraction kit (Yekta Tajhiz Azma, Tehran, Iran) according to the manufacturer's instructions. The partial region of cox 1 gene with approximately 700bp was amplified using the LC01490 (5'GGTCAACAAATCATAAAGATATTGG-3') and HCO2198 (5'-TAAACTTCAGGGTGACCAAAAAATCA3') sequences as cox 1 gene forward and reverse primers (Folmer, Black et al. 1994). The thermal PCR profiles included an initial denaturation step at $95^{\circ} \mathrm{C}$ for 6 minutes followed by 35 cycles of denaturation at $95^{\circ} \mathrm{C}$ for 45 seconds, annealing at $50^{\circ} \mathrm{C}$ for 45 seconds, an initial extension step at $72{ }^{\circ} \mathrm{C}$ for 60 seconds, and a final extension step at $72^{\circ} \mathrm{C}$ for 10 minutes.

\section{Sequencing and phylogenetic analysis}

The PCR products were sequenced using an ABI 3130xl platform (Applied Biosystems, Foster City, California, USA). The sequences identified by $A B I$ system were edited and analyzed by BioEdit software (http://www.mbio.ncsu.edu/bioedit/bioedit.html). The sequences were compared with the sequences deposited in the GenBank database by BLAST program (http://www.ncbi.nlm.nih.gov/). The sequence of Trichostrongylus species deposited in the GenBank database were given the Accession Numbers: MW051252-MW051254 for T. axei; MW051250 and MW051251 for T. colubriformis, and MW051255 and MW051256 for T. vitrinus).

Multiple sequence alignments were conducted with ClustalW incorporated in the BioEdit software. Phylogenetic tree was constructed by the MEGA7 software (Molecular and Evolution Genetic Analysis v7). The maximum likelihood method based on the Tamura 3-parameter model and maximum-likelihood algorithm was applied. Bootstrap value was done based on 1000 replications in the topology of the tree and Dictyocaulus capreolus considered as out group (Fig 2). Pairwise distance comparisons were clarified for seven sequences of Trichostrongylus species isolated in the present study as well as other species with BioEdit software.

\section{Results}

All of the study male worms were identified based on the morphological characteristics of male copulatory spicules and gubernaculum (Fig 1). Trichostrongylus axei was isolated from cattle, sheep and goat but $T$. colubriformis and $T$. vitrines only detected from sheep and goats. The isolates were successfully amplified for Cox 1 gene with specific band. Sequence results confirmed three species of $T$. colubriformis, T. vitrines, and T. axei among the specimens. A dendrogram, based on the phylogenetic analysis, showed that the species were placed along, with the same species, obtained from the GenBank database, into distinct cluster of the tree (Fig 2). The genetic divergence within the species of T. axei, T. 
colubriformis, and T. vitrinus obtained in this study were $0-2.5 \%, 0.77 \%$ and $0 \%$, respectively. Two species of Trichostrongylus including T. axei and T. vitrinus isolated from sheep and goats were quite similar. The intra-species distance rate within the specimens of $T$. axei, T. colubriformis, and T. vitrinus obtained in this study and those available in the GenBank amounted to $0.95-3.1 \%(1.9 \%), 0.19-4.08 \%(2.4 \%)$ and 0 $2.32 \%(1.5 \%)$, respectively.

In this study, the mean inter-species differences between our T. axeispecimens, compared with $T$. colubriformis and $T$. vitrinus isolates, were $14.4 \%$ and $14.6 \%$, respectively. Also, the mean genetic difference between the T. colubriformis specimens, compared with T. vitrinus, was $15.4 \%$.

Based on our sequences and those deposited in the GenBank, the mean inter-species distance rates between the isolates of T. axei and those of T. colubriformis and T. vitrinus were $13.5 \%$ and $14.5 \%$, respectively. Also, the mean genetic diversity between the isolates of T. colubriformis and the isolates of T. vitrinus was $14.9 \%$.

\section{Discussion}

Three species of Trichostrongylus including T. colubriformis, T. vitrinus, and T. axei identified in the present study, along with the data already reported from Iran confirm that the predominant species in herbivorous were T. colubriformis, T. vitrinus, and T. axei [14]. Iran is one of the most important foci for Trichostrongylus infection among human and animal hosts [12, 16, 35, 36]. Proper conditions such as humidity and climate in northern parts of the country including Mazandaran and Guilan provinces lead to permanent establishment of the life cycle process of soil transmitted helminthes in the regions $[2,16,35]$.

In the present study the authors used the sequence analysis protocol for detecting mitochondrial cox 1 gene, whereas several other studies reported from Iran employed ITS-rDNA gene specific for the phylogenetic analysis of Trichostrongy/us species $[2,12,16]$. The nuclear ribosomal gene is widely applied to studies of deep and shallow phylogenetic relationship in the phylum Nematoda $[2,16,37,38]$. Recent studies elustrated that the mitochondrial genes to be the proper options for phylogenomics approach and specifically for the Cox/gene that has mainly been used in population genetic surveys for various nematode parasites of the vertebrates [38, 39, 40].

Several studies have illustrated that the sequence differences between the members of the Trichostrongylus genus is not noticeable based on the ITS2 gene $[2,12,16]$. For example, Ashrafi et al. (2020) reported a mean inter-species distance rate of $2.6 \%$ within different species of Trichostrongylus while in the current study the mean inter-species variation within our specimens and those available in the GenBank was $13.5-14.9 \%$. Due to the high level divergence in the Cox 1 gene, it could be considered as a valuable genetic tool for phylogenetic and taxonomic studies on the members of the Trichostrongylus genus [21]. The phylogenetic tree constructed in our study represented that three species of $T$. colubriformis, $T$. vitrines, and $T$. axei were separated in distinct cluster along with the same species obtained from other studies in different countries (Fig 2). The results of genetic diversity within the 
species showed that the intra-species distance rate among the present isolates was so close, indicating high proximity of the sequences in the region.

Little information on mitochondrial genes of Trichostrongyloidea superfamily is available. Palevich et al. (2020) in New Zealand investigated the complete mitochondrial genomes of $H$. contortus and $T$. circumcincta by phylogenetic analysis [32]. Another study in Uzbekistan was performed based on ribosomal (ITS2) and mitochondrial (Cox1) of Marshallagia sp. the result of the study indicate that ITS2 sequences had little variation, therefor the gene did not allow diagnosing species, while Cox 1 is more verified [31]. Ostertagia trifurcata and Marshallagia marshalli in China were evaluated by phylogenetic analysis of the complete mitochondrial genes. The studies introduced complete mt genome sequence of the nematods as a novel genetic markers for population genetic and molecular epidemiology [28, 33]. Two studies reported from Brazil and Australia evaluated the complete mitochondrial genes of H. placei, T. circumcincta, $T$. vitrines, and $T$. axei and suggested that the phylogenomics approach of mtDNA could be applied as a new genetic marker in phylogenetic analysis and geographic relationships among different isolates in population genetic studies $[25,30]$. Cox 1 and nad 4 genes of $T$. axei were analyzed for population genetic structure of the nematode in USA [29]. However, additional sequence studies, especially the analysis of both nuclear and mitochondrial genes, are needed to provide a comprehensive understanding of genetic variation of Trichostrongylus spp. in endemic areas and other parts of Iran.

In conclusion, our study presented three species of $T$. colubriformis, $T$. vitrines and $T$. axei among sheep, cattle and goats in Guilan province, northern Iran. This study investigated the sequence of mitochondrial Cox 1 gene region and concluded that the genetic diversity of the Cox 1 gene is notable and the gene is suitable for analysis of gene diversity of intra-species distance among helminthes. The scarcity of molecular Cox 1 data within Trichostrongylus spp. in various geographical regions and hosts will be needed to reconstruct the total phylogenetic relationships of this group of nematode. Thus, the findings of the present study suggest that the analysis of complete mitochondrial genome to be the focus of further experiments in the future research.

\section{Declarations}

\section{Acknowledgements}

We would like to appreciate the assistance offered by the colleagues at the Department of Parasitology and Mycology, Medical School, Guilan University of Medical Sciences. The authors are grateful to the abattoir personnel in Talesh, Guilan Province of Iran. We also thank Dr. Ali Asghar Pahlevan for editing the final version of the English manuscript.

\section{Ethics approval and consent to participate}

This study was approved by the Medical Ethics Committee of Guilan University of Medical Sciences (Approval code: IR.GUMS.REC.1397.176). 


\section{Consent for publication}

Not applicable.

\section{Competing interests}

The authors declared no conflict of interests.

\section{Funding}

The project was financially supported by the Deputy for research and technology of Guilan University of Medical Sciences.

\section{Authors' contributions}

Project conceptualization and management: MS: Data collection. Formal analysis. Writing, review and editing. EH: Study design. Data curation. Writing: original draft

\section{Availability of data and material}

The datasets used and/or analysed during the current study are available on reasonable request. Representative nucleotide sequences generated in this study were submitted to the GenBank database under the accession numbers MW051250- MW051256.

\section{References}

1. Roberts L, Janovy J: Foundations of parasitology. 8th edn. 701 pp. New York, McGraw-Hill; 2009.

2. Sharifdini M, Heidari Z, Hesari Z, Vatandoost S, Kia EB. Molecular phylogenetics of Trichostrongylus species (Nematoda: Trichostrongylidae) from humans of Mazandaran province, Iran. The Korean Journal of Parasitology. 2017;55 3:279.

3. Ghanbarzadeh L, Saraei M, Kia E, Amini F, Sharifdini M. Clinical and haematological characteristics of human trichostrongyliasis. J Helminthol. 2018;93:149-53.

4. Wall EC, Bhatnagar N, Watson J, Doherty T. An unusual case of hypereosinophilia and abdominal pain: an outbreak of Trichostrongylus imported from New Zealand. Journal of travel medicine. 2011;18 1:59-60.

5. da Rocha LO, da Silva Lemos GC, Vieira IJC, Braz-Filho R, de Paiva Freitas S, Glória LS, et al. Chemical characterization and in vitro biological activity of Cymbopogon citratus extracts against Haemonchus spp. and Trichostrongylus spp. nematodes from sheep. Parasitology. 2020;147 13:1559-68.

6. McLeod R. Costs of major parasites to the Australian livestock industries. International journal for parasitology. 1995;25 11:1363-7. 
7. Phosuk I, Intapan PM, Sanpool O, Janwan P, Thanchomnang T, Sawanyawisuth K, et al. Molecular evidence of Trichostrongylus colubriformis and Trichostrongylus axei infections in humans from Thailand and Lao PDR. The American journal of tropical medicine and hygiene. 2013;89 2:376-9.

8. Ghadirian E. Human infection with Trichostrongylus lerouxi (Biocca, Chabaud, and Ghadirian, 1974) in Iran. The American journal of tropical medicine and hygiene. 1977;26 6:1212-3.

9. Ghadirian E, Arfaa F, Sadighian A. Human infection with Trichostrongylus capricola in Iran. The American journal of tropical medicine and hygiene. 1974;23 5:1002-3.

10. Ghasemikhah R, Mirhendi H, Kia E, Mowlavi G, Sarmadian H, Meshgi B, et al. Morphological and morphometrical description of Trichostrongylus species isolated from domestic ruminants in Khuzestan province, southwest Iran. Iranian journal of parasitology. 2011;6 3:82.

11. Shahbazi A, Fallah E, Koshki MK, Nematollahi A, Ghazanchaei A, Asfaram S. Morphological characterization of the Trichostrongylus species isolated from sheep in Tabriz, Iran. Research Opinions in Animal and Veterinary Sciences. 2012;2 5:309-12.

12. Ashrafi K, Sharifdini M, Heidari Z, Rahmati B, Kia EB. Zoonotic transmission of Teladorsagia circumcincta and Trichostrongylus species in Guilan province, northern Iran: molecular and morphological characterizations. BMC Infectious Diseases. 2020;20 1:1-9.

13. Ashrafi K, Tahbaz A, Sharifdini M, Mas-Coma S. Familial Trichostrongylus infection misdiagnosed as acute fascioliasis. Emerging infectious diseases. 2015;21 10:1869.

14. Ghadirian E, Arfaa F. Present status of trichostrongyliasis in Iran. The American journal of tropical medicine and hygiene. 1975;24 6:935-41.

15. Sharifdini M, Ghanbarzadeh L, Barikani A, Saraei M. Prevalence of intestinal parasites among rural inhabitants of Fouman, Guilan Province, Northern Iran with emphasis on Strongyloides stercoralis. Iranian Journal of Parasitology. 2020;15 1:91.

16. Sharifdini M, Derakhshani S, Alizadeh SA, Ghanbarzadeh L, Mirjalali H, Mobedi I, et al. Molecular identification and phylogenetic analysis of human Trichostrongylus species from an endemic area of Iran. Acta tropica. 2017;176:293-9.

17. Gholami S, Babamahmoodi F, Abedian R, Sharif M, Shahbazi A, Pagheh A, et al. Trichostrongylus colubriformis: possible most common cause of human infection in Mazandaran province, North of Iran. Iranian journal of parasitology. 2015;10 1:110.

18. Anvari-Tafti M, Sazmand A, Hekmatimoghaddam S, Moobedi I. Gastrointestinal helminths of camels (Camelus dromedarius) in center of Iran. Trop Biomed. 2013;30 1:56-61.

19. Borji H, RAZMI GR, MOVASAGHI A, NAGHIBI AA, MALEKI M. SHORT PAPER: A STUDY ON GASTROINTESTINAL HELMINTHS OF CAMELS IN MASHHAD ABATTOIR, IRAN. 2010.

20. Biocca E, Chabaud A, Ghadirian E. Trichostrongylus lerouxi n. sp., parasite of Bos taurus. Parassitologia. 1974;16 2-3:199.

21. Ghasemikhah R, Sharbatkhori M, Mobedi I, Kia E, Harandi MF, Mirhendi H. Sequence analysis of the second internal transcribed spacer (ITS2) region of rDNA for species identification of 
Trichostrongylus nematodes isolated from domestic livestock in Iran. Iranian journal of parasitology. 2012;7 2:40.

22. de Bellocq JG, Ferte H, Depaquit J, Justine J-L, Tillier A, Durette-Desset M-C. Phylogeny of the Trichostrongylina (Nematoda) inferred from 28S rDNA sequences. Molecular Phylogenetics and Evolution. 2001;19 3:430-42.

23. Hoberg EP, Monsen KJ, Kutz S, Blouin MS. Structure, biodiversity, and historical biogeography of nematode faunas in holarctic ruminants: morphological and molecular diagnoses for Teladorsagia boreoarcticus n. sp.(Nematoda: Ostertagiinae), a dimorphic cryptic species in muskoxen (Ovibos moschatus). The Journal of parasitology. 1999:910-34.

24. von Samson-Himmelstjerna G, Harder A, Schnieder T. Quantitative analysis of ITS2 sequences in trichostrongyle parasites. International Journal for Parasitology. 2002;32 12:1529-35.

25. dos Santos LL, Prosdocimi F, Lima NCB, da Costa IR, Cardoso DC, Drummond MG, et al. Comparative genomics and phylogenomics of Trichostrongyloidea mitochondria reveal insights for molecular diagnosis and evolutionary biology of nematode worms. Gene Reports. 2017;9:65-73.

26. Hu M, Chilton NB, Gasser RB. The mitochondrial genomics of parasitic nematodes of socioeconomic importance: recent progress, and implications for population genetics and systematics. Advances in parasitology. 2004;56:134-213.

27. Saccone C, De Giorgi C, Gissi C, Pesole G, Reyes A. Evolutionary genomics in Metazoa: the mitochondrial DNA as a model system. Gene. 1999;238 1:195-209.

28. Ahmad AA, Yang X, Zhang T, Wang C, Zhou C, Yan X, et al. Characterization of the complete mitochondrial genome of Ostertagia trifurcata of small ruminants and its phylogenetic associations for the Trichostrongyloidea superfamily. Genes. 2019;10 2:107.

29. Archie EA, Ezenwa VO. Population genetic structure and history of a generalist parasite infecting multiple sympatric host species. International journal for parasitology. 2011;41 1:89-98.

30. Jex AR, Hall RS, Littlewood DTJ, Gasser RB. An integrated pipeline for next-generation sequencing and annotation of mitochondrial genomes. Nucleic Acids Research. 2010;38 2:522-33.

31. Kuchboev A, Sobirova K, Karimova R, Amirov O, von Samson-Himmelstjerna G, Krücken J. Molecular analysis of polymorphic species of the genus Marshallagia (Nematoda: Ostertagiinae). Parasites \& vectors. 2020;13 1:1-12.

32. Palevich N, Maclean PH, Choi Y-J, Mitreva M. Characterization of the complete mitochondrial genomes of two sibling species of parasitic roundworms, Haemonchus contortus and Teladorsagia circumcincta. 2020.

33. Sun M-M, Han L, Zhang F-K, Zhou D-H, Wang S-Q, Ma J, et al. Characterization of the complete mitochondrial genome of Marshallagia marshalli and phylogenetic implications for the superfamily Trichostrongyloidea. Parasitology research. 2018;117 1:307-13.

34. Barghandan T, Hajialilo E, Sharifdini M, Javadi A. Prevalence and phylogenetic analysis of gastrointestinal helminths (Nematoda: Trichostrongylidae) in ruminant livestock of northwest Iran. Ankara Üniversitesi Veteriner Fakültesi Dergisi. 2019;67 1:65-72. 
35. Alemi A, Arfaa F. Prevalence of intestinal helminthiasis in the rural area of gilan province (caspian littoral). Iranian Journal of Public Health. 1978:25-34.

36. Massoud J, Arfaa F, Jalali H, Keyvan S. Prevalence of intestinal helminths in Khuzestan, Southwest Iran, 1977. The American Journal of Tropical Medicine and Hygiene. 1980;29 3:389-92.

37. Holterman $M$, van der Wurff $A$, van den Elsen $S$, van Megen $H$, Bongers $T$, Holovachov $O$, et al. Phylum-wide analysis of SSU rDNA reveals deep phylogenetic relationships among nematodes and accelerated evolution toward crown clades. Molecular biology and evolution. 2006;23 9:1792-800.

38. Kiewnick S, Holterman M, van den Elsen S, van Megen H, Frey JE, Helder J. Comparison of two short DNA barcoding loci (COI and COII) and two longer ribosomal DNA genes (SSU \& LSU rRNA) for specimen identification among quarantine root-knot nematodes (Meloidogyne spp.) and their close relatives. European Journal of Plant Pathology. 2014;140 1:97-110.

39. Nadler SA, Hudspeth DS. Phylogeny of the Ascaridoidea (Nematoda: Ascaridida) based on three genes and morphology: hypotheses of structural and sequence evolution. Journal of Parasitology. 2000;86 2:380-93.

40. Otranto D, Testini G, De Luca F, Hu M, Shamsi S, Gasser R. Analysis of genetic variability within Thelazia callipaeda (Nematoda: Thelazioidea) from Europe and Asia by sequencing and mutation scanning of the mitochondrial cytochrome $c$ oxidase subunit 1 gene. Molecular and cellular probes. 2005;19 5:306-13.

\section{Figures}




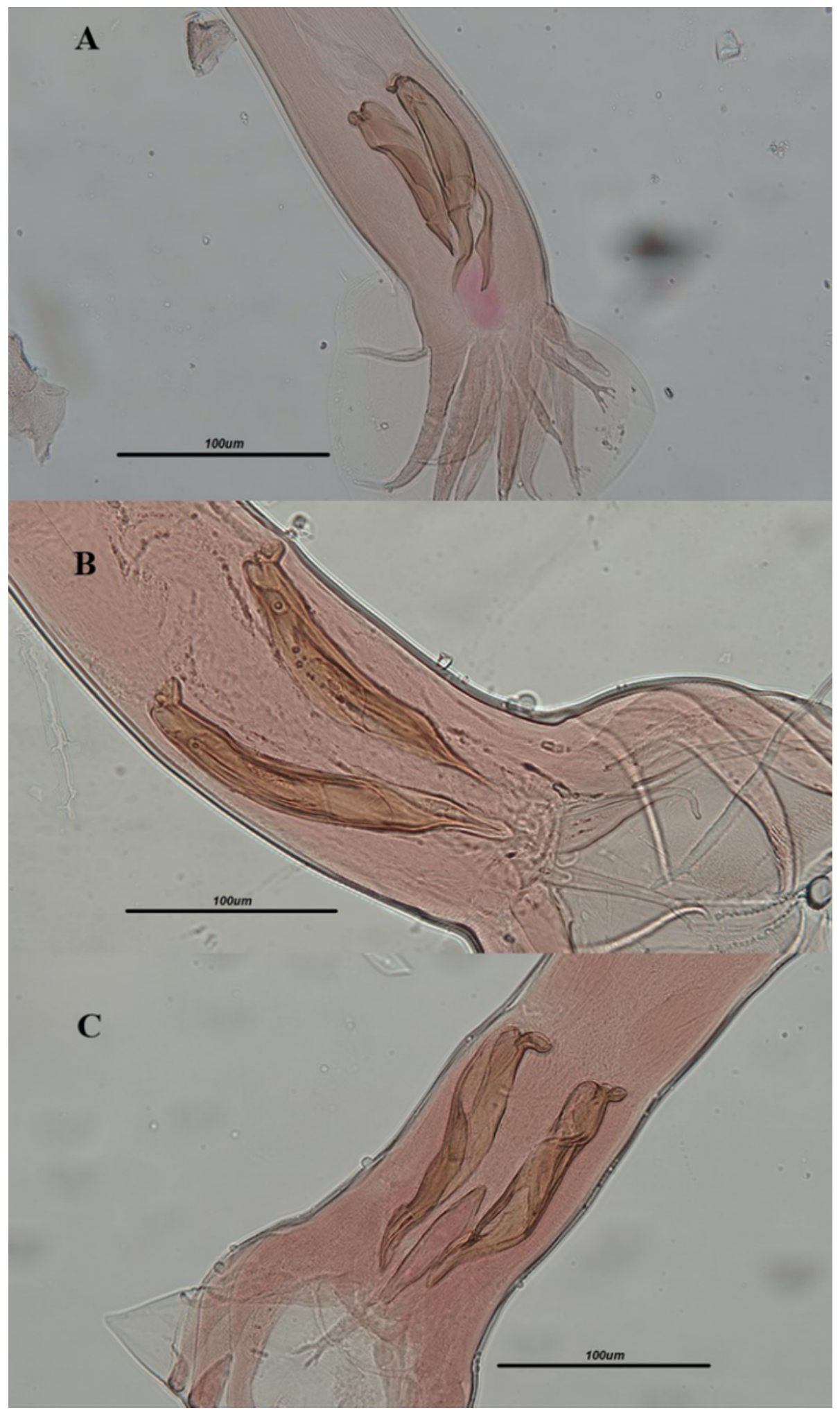

\section{Figure 1}

Copulatory bursa and spicules of T. axei (A), T. vitrines (B) and T. colubriformis (C) 


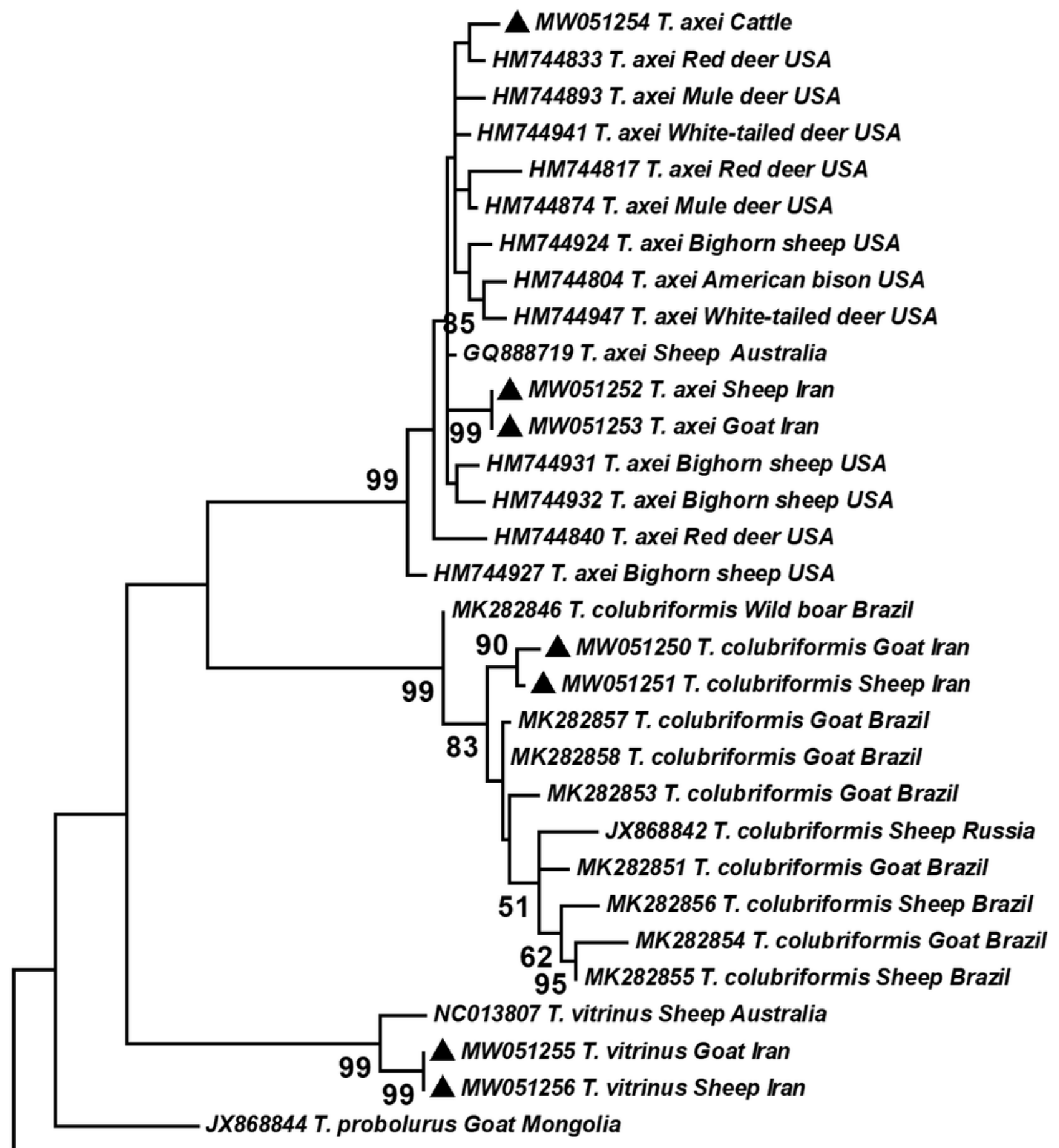

KR231673 Dictyocaulus capreolus

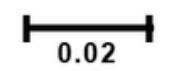

Figure 2

Phylogenetic tree of isolates of Trichostrongylus spp. obtained in this study $(\boldsymbol{\Lambda})$ and other isolates of Trichostrongylus retrieved from GenBank based on cox 1 gene. The tree was designed by using the Maximum-Likelihood test and the Tamura 3-parameter model as implemented in the MEGA7 software. Dictyocaulus capreolus was used as an out group. 


\section{Supplementary Files}

This is a list of supplementary files associated with this preprint. Click to download.

- graphicalabstract.jpg 